\title{
An Intrinsic Source of Behavioral Regulation That Influences Discrete Responses to Cues Important for the Initiation of Suckling
}

\author{
Leigh F. Bacher \\ Binghamton University
}

\author{
Steven S. Robertson \\ Cornell University
}

\author{
William P. Smotherman \\ Binghamton University
}

\begin{abstract}
The present experiment investigated the relationship between motor activity and oral grasping of an artificial nipple in newborn rats. Pups orally grasped the artificial nipple, and they performed more and longer oral grasps in the latter portion of the nipple presentation. Motor activity was cyclical, and this cyclicity was evident before and during presentation of the artificial nipple. The onset of an oral grasp response was preceded by a period of relatively low motor activity, and the termination of a grasp was followed by relatively high motor activity. The newborn rat pup's intrinsic oscillations in motor activity may regulate the expression of discrete responses to cues important for the initiation of suckling.
\end{abstract}

Late-gestation fetal rats respond to suckling stimuli including milk and an artificial nipple (AN) with species-typical behavioral patterns such as the stretch response to milk (Robinson \& Smotherman, 1992, 1994) and oral grasping of an AN (Robinson, Hoeltzel, Cooke, Umphress, Murrish, \& Smotherman, 1992). Not only do these fetal responses highlight that the origins of appetitive behaviors of the neonate begin in utero, but they also raise new questions about postnatal behavior. In the present study, we investigate the relationship between neonatal rats' responsiveness to sucking stimuli and spontaneous motor activity that was recently described in the late-gestation fetal rat (Reilly, Robertson, MacLennan, \& Smotherman, 1997).

The oral responses of late-gestation fetal rats to suckling stimuli occur on a background of spontaneous motor activity that fluctuates on a time scale of approximately $1 \mathrm{~min}$ (Reilly et al., 1997; Smotherman, Robinson, \& Robertson, 1988). These fluctuations, known as cyclic motor activity, are persistent and irregular oscillations in general body activity. Cyclic motor activity has been observed in a variety of species of young animals such as fetal sheep (Robertson \& Bacher, 1995), prehatchling chicks (Hamburger, Balaban, Oppenheim, \& Wenger, 1966), and fetal and infant humans (Robertson, 1982, 1985).

Leigh F. Bacher and William P. Smotherman, Department of Psychology, Binghamton University; Steven S. Robertson, Department of Human Development, Cornell University.

This research was supported by National Institutes of Health, National Institute of Child and Human Development Grants HD23814 and HD28014, and by Merit Award HD16102. We thank Holly Spanier and Sandy Yip for help with data collection.

Correspondence concerning this article should be addressed to Leigh $F$. Bacher, Department of Psychology, Binghamton University, Vestal Parkway East, Binghamton, New York 13902-6000. Electronic mail may be sent to lbacher@binghamton.edu.
Fetuses' oral grasp responses to an AN may be regulated by these intrinsic oscillations in motor activity (Reilly et al., 1997). In these studies, oral grasping of the AN was related to features of motor activity at the time of nipple presentation as well as the recent history of motor activity. Specifically, fetuses were more likely to grasp the nipple when movement amplitude was both low at the time of nipple presentation and more variable during the 30-s period preceding nipple presentation (MacLennan, Smotherman, \& Robertson, 1998). This suggests that characteristics of motor activity modulate fetuses' responsiveness to external stimulation.

The results of the fetal studies raise at least two major questions. Does motor activity of the newborn rat show cyclic temporal organization? Is a similar relationship between motor activity and oral grasping observed in the newborn as in the fetus? Although the motor activity of infant rats has been described as phasic (Corner, 1977, 1978), cyclic organization in the newborn rat has not been quantified. Temporal structure also has been noted in infant rats when huddling. Changes in ambient temperature result in changes in the direction of pup movement and huddle surface area (Alberts, 1978). In the context of suckling, motor activity of the neonatal rat follows a highly structured sequence of head and body movements during nipple search and attachment to the maternal nipple (Eilam \& Smotherman, 1998). These studies suggest that temporal structure is characteristic of infant rat behavior (for the individual and the group) and that an exploration of the temporal relationship between motor activity and early suckling may be informative.

In the present experiment, we investigated the relationship between oral grasping of an AN and ongoing motor activity in newborm rats. The main objectives of the experiment were to examine newborn rats' general motor behavior for evidence of cyclic motor activity and to identify and describe the temporal relationship between motor activity and the expression of oral grasp responses during newborns' first experiences with an AN. To meet these objectives, motor activity and oral grasp responses 
were recorded in 18 newborn rat pups delivered by cesarean section and tested before any interaction with the mother. Cesarean section permits testing before pups have any suckling experience and therefore permits the observation of newborns' first experiences with extra-uterine stimuli (Smotherman, Goffman, Petrov, \& Varlinskaya, 1997; Smotherman, Petrov, \& Varlinskaya, 1997). During the first $10 \mathrm{~min}$ of a 20 -min observation period, pups were observed without any other manipulation to provide information about baseline levels of motor activity. During the second $10 \mathrm{~min}$, an AN was presented continuously to the rat pup.

Investigation into the temporal patterning of behavior of the newborn may reveal information about the effects of intrinsic oscillations on responsiveness to environmental stimuli as well as the manner in which discrete behaviors (e.g., oral grasp response) are integrated with ongoing cycles of movement. Furthermore, the results of this study have implications for greater understanding of behavior during newborn pups' first suckling episode because the oral grasp response is an important component of the suckling sequence (Smotherman, Goffman, et al., 1997).

\section{General Method}

\section{Subjects}

Newborn subjects ( $n=18$; 9 females) were the offspring of SpragueDawley rats (from Charles River Laboratories, Wilmington, MA) produced by time-matings. For a 4 -day breeding period, adult rats were housed in groups of three females and one male in plastic breeding cages $(36 \times$ $47 \times 20 \mathrm{~cm}$ ). Each day, vaginal smears were collected. The first day of detectable sperm was designated as Embryonic Day 0 (E0). Birth occurs on E21.5; the day of birth was designated Postnatal Day 0 (P0). Pregnant female rats were maintained at constant room temperature $\left(22^{\circ} \mathrm{C}\right)$, on a 12-hr light-dark cycle (lights on at 0700) until the day of testing. Food and water were available ad libitum. Rats used in these experiments were treated in accordance with guidelines for animal care established by the National Institutes of Health (1986).

\section{Cesarean Delivery}

To control the type and quantity of stimulation the pups received between delivery and testing, experimental subjects were delivered by cesarean section near term on E21. Pups delivered vaginally that have $24 \mathrm{hr}$ of maternal experience behave similarly toward an AN as do pups delivered by cesarean section that have no maternal experience (Petrov, Varlinskaya, \& Smotherman, 1997).

After brief ether anesthesia, the pregnant rat was given an injection of $100 \%$ ethanol $(100 \mu \mathrm{l})$ between the first and second lumbar vertebrae to block neural transmission in the spinal cord, which eliminates sensation in the lower part of the body. With this method, neither ether nor ethanol affects pups' behavior during the time the pups are tested after birth. A midline laparotomy provided direct access to the uterine horns. Fetuses were removed 1 at a time. Umbilical circulation was halted by ligation of the umbilical cord with 6.0 surgical suture. The umbilical cord was then cut on the placental side of the ligation. Pups from an individual female were placed together in a $12 \mathrm{~cm}$ long $\times 12 \mathrm{~cm}$ wide $\times 6 \mathrm{~cm}$ high plastic container lined with a water-moistened paper towel. The container with newborn pups was put in a temperature-regulated $\left(33^{\circ} \mathrm{C}\right)$ incubator. When the cesarean section procedure was completed, the donor female was killed by rapid cervical dislocation.

\section{Procedure}

Before testing, each pup was transported from the incubator to the testing site and allowed to acclimate to the testing environment for 5 min.
For each pup, testing followed the same sequence: 10 min of movement data were collected during which the pup was unstimulated (baseline), followed immediately by 10 min of continuous AN presentation. Pups were tested between 3.2 and 5.5 hours $(M=4.1 \pm .61)$ after cesarean delivery. To avoid potential litter effects through overrepresentation from a single litter, not more than 1 male and 1 female were tested from a given litter (Holson \& Pearce, 1992)

Presentation of the AN. The AN was sculpted from soft vinyl material so that its dimensions were approximately $16 \mathrm{~mm}$ in length, tapered to a. diameter of $1 \mathrm{~mm}$ at its rounded tip (Robinson et al., 1992). A ring (7 mm in diameter) was made from the same material and secured $7 \mathrm{~mm}$ from the tip of the nipple. This ring provided a point of contact for the snout of the pup when it made an oral grasp of the nipple. The nipple was fixed to the end of a dental probe that enabled the experimenter to position the nipple. During continuous presentation, the experimenter kept the AN in contact with the pup's perioral area. This contact was very gentle; the tip of the AN was not forced into the pup's mouth (Petrov, Varlinskaya, \& Smotherman, 1997; Smotheman, Goffman, et al., 1997). Presenting the AN to the pup did not produce detectable artifacts in the output of the movement sensor.

Behavioral observation. Testing occurred in a $50.0 \mathrm{~cm} \times 63.5$ $\mathrm{cm} \times 26 \mathrm{~cm}$ transparent testing box. The box was constructed with two holes in one side for the experimenter to present the AN. Each pup was placed on a movement sensor that was surrounded by a fixed plastic border ( $7.5 \mathrm{~cm}$ long $\times 7.5 \mathrm{~cm}$ wide $\times 2 \mathrm{~cm}$ high) to keep the pup on the sensor. The temperature was kept between 34 and $37{ }^{\circ} \mathrm{C}$ by heating pads in the testing box. A shallow dish of warm water was placed in the heated testing box to maintain humidity levels. So that subsequent subjects could not detect signs (odors, fluids) of previously tested pups, the film covering the movement sensor and the plastic border was changed between pups.

Measurement of gross motor activity. Motor activity was measured at $60 \mathrm{~Hz}$ with a piezoelectric speaker element as a movement sensor (Archer Model 273-091, Radio Shack). When the element (a 4-cm diameter, thin metal disk) was deformed by the pup's movement, small voltages were generated. Thresholds were applied to the raw movement signal to remove low amplitude background activity caused by respiration, heartbeat, or electrical noise. A single threshold was selected and used for all pups. The movement data were rectified and integrated to create movement-time series of 1 or $30 \mathrm{~Hz}$ depending on the analysis. These techniques have been used reliably in previous studies (MacLennan et al., 1998; Reilly et al., 1997).

Oral grasping of the AN. Oral grasping of the AN by the newborn rat is a highly stereotyped, easily identifiable behavior. The pup's head moves quickly toward the AN, its mouth opens and then closes around the tip of the AN, pressure is applied on the AN by the jaws, a seal is formed around the tip of the nipple, and negative pressure is exerted (Robinson et al., 1992). In the present experiment, the experimenter noted oral grasp responses by depressing a switch at the onset of the grasp and releasing the switch at its offset. This signal was sampled concurrently with the movement signal.

To correct for the reaction time associated with the use of a manual switch, the signal marking the onset and offset of the grasp was shifted $1 / 3 \mathrm{~s}$ earlier. This estimate of reaction time for moving a manual switch was based on reaction times from pilot testing of a similar task and reaction times published in research using similar manual tasks (Chelazzi, Biscaldi, Corbetta, Peru, Tassinari, \& Berlucchi, 1995; Gomez, Millan, Atienza, Aguilar-Bravo, Vazquez \& Delinte, 1998).

Analyses. The first set of analyses described the general characteristics of motor activity over many minutes during both the baseline and nipple presentation periods and also described the number and duration of oral grasp responses observed during nipple presentation. Oral grasping behavior and motor activity were also analyzed for changes during the nipple presentation period that might indicate how pups' responsiveness to the AN changes over the first minutes of experience with this stimulus. The second set of analyses focused on short term (scale of seconds) relation- 
ships between motor activity and the expression of oral grasp responses. Motor activity before, during, and after oral grasps was analyzed.

Preliminary analyses testing for sex differences were conducted. No sex differences were found in any primary dependent variable.

\section{Analysis 1: Characteristics of Motor Activity and Oral Grasp Responses}

The first set of analyses describes several characteristics of motor activity and the expression of oral grasp responses. The level, temporal variability, and cyclic organization of motor activity were analyzed during the $10-$ min baseline and AN presentation periods. In addition, changes in the level and variability of motor activity during AN presentation were analyzed. The number and duration of oral grasp responses during $\mathrm{AN}$ presentation also were assessed.

\section{Motor Activity}

Method. The level of motor activity during each period of the experiment (baseline and AN presentation) was measured by taking the average of the movement samples in the $1-\mathrm{Hz}$ movement-time series. The measure of temporal variability was the average absolute difference between successive movement samples at $1 \mathrm{~Hz}$. This measure of variability better reflects moment-to-moment changes in movement than does, for example, the standard deviation (MacLennan et al., 1998; Reilly et al., 1997).

For each 2-min interval during the AN presentation, the average level of motor activity and the temporal variability of motor activity were calculated for each pup. The level and variability of motor activity across pups for each of these five intervals were compared by using one-way repeated measures analyses of variance (ANOVAs). Scheffe's post hoc tests (Winer, 1971) were used to clarify the pattern of differences $(p<.05)$.

Spectral analysis was conducted on movement-time series from the baseline and AN presentation periods to identify and quantify cyclic organization. The $30-\mathrm{Hz}$ movement-time series were first integrated to 1 $\mathrm{Hz}$. Each motor activity time series was detrended and the Fourier transform of the auto correlation function was calculated with an algorithm written by Jenkins and Watts (1968). When calculated this way, the movement spectrum represents stable estimates of the relative strength of periodic fluctuations occurring at different frequencies. The area under the movement spectrum in a given frequency band represents the proportion of movement variance between the two frequencies that define that band. Cyclic organization was inferred from a peak in the movement spectrum that exceeded the upper $99 \%$ confidence limit of white noise. This analysis has been used in previous investigations of cyclic organization of spontaneous motor activity (MacLennan et al., 1998; Reilly et al., 1997; Robertson, 1985; Smotherman, Robinson \& Robertson, 1988).

Four measures of cyclic organization were calculated for each spectrum that contained a significant peak: frequency of the largest peak in the spectrum, height of the largest peak in the spectrum, width of the largest peak in the spectrum, and proportion of variance due to fluctuations greater than three cycles per minute. The frequency indicates the dominant rate of oscillation in motor activity, the height measures the strength of the dominant oscillation, and the width indicates the irregularity of the dominant oscillation. The fourth measure was used to capture relatively high frequency fluctuations across a large frequency band. The proportion of variance was calculated by computing the area under the activity spectrum above three cycles per minute. The six measures of motor activity (level, variability, and the four measures of cyclic organization) for baseline and AN presentation periods were compared by using paired $t$ tests.

Results. Most of the features of motor activity did not change during presentation of the $\mathrm{AN}$. The level and temporal variability of motor activity did not change from baseline to AN presentation
Table 1

Means ( $\pm S D$ ) for Motor Activity Measures During Baseline and AN Presentation Periods

\begin{tabular}{lcccc}
\hline \multicolumn{1}{c}{ Measure } & Baseline & AN presentation & $t(17)$ & $p$ \\
\hline Level & $2.38 \pm 1.33$ & $2.58 \pm 1.20$ & -0.95 & .35 \\
Variability & $1.68 \pm 0.79$ & $1.67 \pm 0.66$ & -0.33 & .74 \\
\% Pups with CM & 100 & 100 & - & - \\
Frequency & $0.695 \pm 0.31$ & $0.877 \pm 0.39$ & 1.56 & .14 \\
Strength & $0.474 \pm 0.17$ & $0.373 \pm 0.13$ & 2.35 & .03 \\
Irregularity & $0.644 \pm 0.21$ & $0.630 \pm 0.06$ & 0.01 & .99 \\
$\%>3$ cpm & $53.9 \pm 0.08$ & $56.6 \pm 0.06$ & -2.27 & .04 \\
\hline
\end{tabular}

Note. Dashes indicate data not applicable. $\mathrm{AN}=$ artificial nipple; $\mathrm{CM}=$ cyclic motor activity; $\mathrm{cpm}=$ cycles per minute.

(see Table 1). Neither the average level of motor activity nor the temporal variability in motor activity in each 2-min interval changed over the 10-min AN presentation period, $F(4,68)=2.18$, $p=.08$, and $F(4,68)=2.19, p=.08$, respectively.

Evidence of cyclic organization in motor activity was present in the movement time series of each pup during both the baseline and the AN presentation periods. The frequency of the dominant peak, $t(17)=1.56, p=.14$, and the irregularity (width) of the dominant peak, $t(17)=.01, p=.99$, did not change. However, the strength of the dominant peak decreased from baseline during AN presentation, $t(17)=2.35, p=.03$, and the proportion of variance due to fluctuations greater than three cycles per minute increased during AN presentation, $t(17)=-2.27, p=.036$ (Table 1).

Discussion. The level and variability of motor activity in the newborn rat, as well as the frequency and irregularity of the dominant oscillation, were similar during baseline and AN presentation. However, strength of the dominant oscillation decreased and the relative power of high frequency oscillations increased during AN presentation. Although most features of motor activity were unchanged during AN presentation, the presence of several changes in motor activity during AN presentation suggests that pups showed some sensitivity to the perioral stimulation.

Similar results have been reported with the fetal rat (Reilly et al., 1997). During the presentation of the AN, motor activity of the fetal rat showed a decrease in the strength of the dominant oscillation and an increase in the proportion of variance due to fluctuations greater than three cycles per minute. However, unlike the newborn rat, motor activity in fetuses increased, was more variable, and showed more rapid oscillations during AN presentation.

\section{Oral Grasp Responses}

Method. Oral grasp responses selected for the analyses met two criteria. First, the grasps were 1 s or longer in duration. Second, selected grasps could not be preceded or followed by another grasp within $10 \mathrm{~s}$. The duration criterion was used to select grasps that were long enough for an analysis of motor activity during grasping. The criterion for time between grasps permitted the study of body movement before and after oral grasp responses without selecting portions of body movement that occurred in proximity to another grasp.

A one-way, repeated-measures ANOVA was used to compare the number of oral grasp responses over successive 2-min intervals during the 10-min AN presentation period. For the analysis of grasp durations, repeated measures ANOVA was not used because several pups did not grasp during every interval. However, 13 pups grasped during the first two 
intervals and during the last two intervals. A binomial test was used to test whether the longest grasp occurred during the early versus the latter part of the AN presentation.

Results. The number of oral grasp responses observed was 216. From these, grasps that met the selection criteria for the minimum grasp duration ( $1 \mathrm{~s}$ ) and minimum time between grasps (10 s) were identified for further analysis. The number of oral grasp responses that met the selection criteria, $n=102$, represented $45 \%$ of all grasps observed. The percentage of grasps that met the duration criterion was $78.1 \%$, and the percentage of grasps that met the time between grasps criterion was $77.2 \%$.

Every pup grasped the AN. The mean number of oral grasp responses per pup was $5.67(S D=3.0)$. The frequency of oral grasp responses changed over the presentation period, $F(4$, $68)=3.41, p=.013$. The means $( \pm S D)$ for each of the five intervals were: $0.5 \pm 0.9,1.4 \pm 1.4,1.1 \pm 1.2,1.0 \pm 0.8$, and $1.6 \pm 1.1$. Post hoc tests revealed that significantly more grasps were observed in the last 2 min than were observed in the first $2 \mathrm{~min}$.

For the 13 pups that grasped during both the first and the last two intervals, the longest grasp was more likely to occur during the last two intervals $(n=9)$ than during the first $(n=2)$, (binomial $p<.05$; see Figure 1). The number of times the longest grasp was observed in each of the five 2 -min intervals were: $1,1,2,3$, and 6 .

Discussion. The number of oral grasp responses was relatively low during the first $2 \mathrm{~min}$ and highest during the last $2 \mathrm{~min}$. The absence of a decrease in the number of oral grasp responses over time indicates that the pup did not habituate to the AN during continued presentation. The AN reliably elicited oral grasp responses after many minutes of continuous perioral stimulation.

The longest oral grasp responses occurred in the latter portion of the AN presentation period. Increases in the duration of oral grasp responses could occur for one or more reasons. Increased grasp duration over time might reflect cumulative effects of continuous perioral stimulation, oral grasping experience, increased sensitivity to the $\mathrm{AN}$, increased motivation to grasp (deprivation), or rapid maturational change. Furthermore, because the level of pups' motor activity did not change during the AN presentation period, it is not likely that systematic changes in the level of movement contributed to longer grasp durations. Regardless of the mechanism underlying increases in the duration of oral grasp responses,

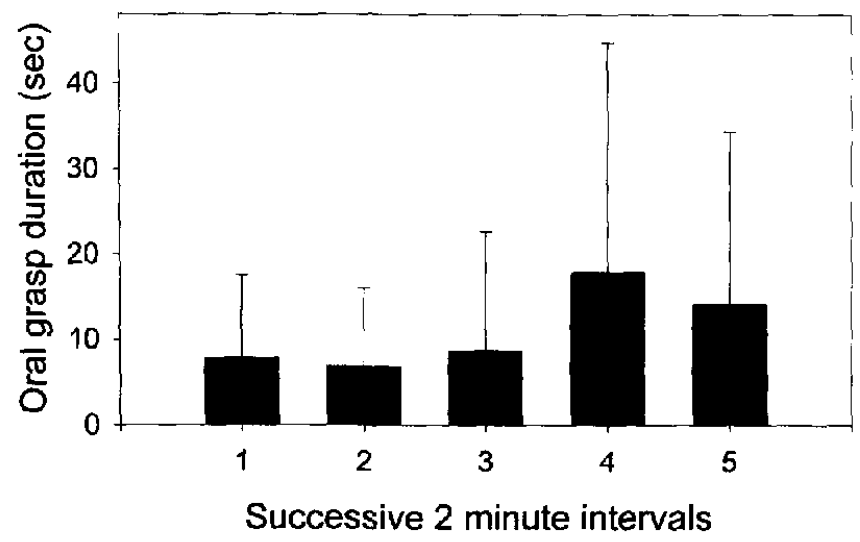

Figure 1. Mean ( $\pm S D$ ) duration of oral grasp responses during each 2-min interval of the artificial nipple presentation period. changes in the duration of oral grasp responses occurred within 10 min after pups' first experiences with the nipple. Such a response may have a consequence of ensuring that the pup is attached to a nipple when the first milk letdown occurs.

\section{Analysis 2: Temporal Relationship Between Fluctuations in Motor Activity and Oral Grasp Responses}

Whereas the first set of analyses measured motor activity over many minutes, the second set of analyses focused on short-term (in seconds) features of motor activity by examining the level and temporal variability of motor activity immediately before, during, and after individual oral grasp responses. In addition, motor activity during the oral grasp response was divided into early, middle, and late segments and was analyzed. These analyses provided information about whether the onset and offset of oral grasp responses were temporally associated with patterns of motor activity.

\section{Method}

The levels of motor activity before, during, and after criterion oral grasp responses were compared by using the $30-\mathrm{Hz}$ movement-time series. The before and after segments were $5 \mathrm{~s}$ long. Because oral grasp responses were of different durations, the movement sample for the during segment of each oral grasp varied in length. The mean levels of motor activity for the before, during, and after segments were compared in a one-way, repeatedmeasures ANOVA. Also, the level of motor activity from the 10-min baseline period was compared with the before, during, and after segments by paired $t$ tests. The same statistical comparisons were made for the measure of temporal variability in motor activity.

A separate analysis of motor activity during oral grasp responses was conducted. Motor activity during each oral grasp was divided into equal thirds (early, middle, and late) that permitted the analysis of changes in movement during the oral grasp. The $30-\mathrm{Hz}$ movement-time series were used in these analyses. One-way, repeated-measures ANOVAs were used to detect differences in the means of the two motor activity measures across the early, middle and late thirds of oral grasp responses.

\section{Results}

The level of motor activity varied systematically before, during, and after oral grasp responses, $F(2,34)=9.0, p=.0008$ (see Figure 2). Post hoc comparisons indicated that motor activity before and during the grasp were lower than motor activity after the grasp. Motor activity during the grasp did not differ from motor activity before the grasp.

Also, the temporal variability of motor activity varied systematically before, during, and after oral grasp responses, $F(2$, 34 ) $=9.17, p=.0015$ (Figure 2). Post hoc comparisons indicated that motor activity before the grasp was less variable than motor activity after the grasp. The variability of movement during the oral grasp did not differ from the variability of motor activity before or after the grasp.

Motor activity during the baseline period was compared with the before, during, and after segments (Figure 2). The level of movement in the before segment was lower than that in the baseline period, $t(17)=2.30, p=.034$. The level of movement in the during segment did not differ from baseline, $t(17)=-.21, p=$ .83. The level of movement in the after segment was greater than the level observed in the baseline period, $t(17)=-2.54, p=.021$. 

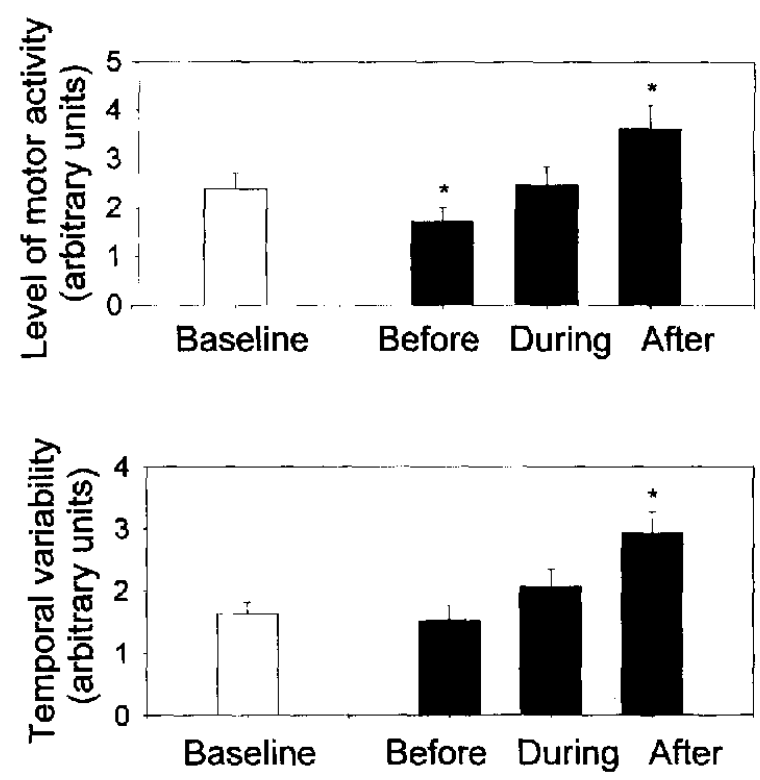

Figure 2. Level of motor activity (top) and variability of motor activity (bottom) during baseline (open bar) and before, during, and after (solid bars) segments around oral grasp responses. Asterisks mark significant differences from baseline. Error bars indicate standard errors.

The temporal variability of movement in the after segment differed from baseline levels, $t(17)=-3.8, p=.0015$. However, movement variability in the before and during segments did not differ from baseline, $t(17)=-1.8, p=.09$, and $t(17)=.50, p=.63$, respectively.

The level of motor activity within the during segment of the oral grasp response also changed systematically, $F(2,34)=4.1, p=$ .025 (Figure 3). Post hoc comparisons indicated that the level of movement in the last third of the grasp was higher than the level of movement in the middle third. Similarly, the temporal variability of motor activity changed during the oral grasp response, $F(2$, 17) $=6.7, p=.003$. Post hoc comparisons indicated that the variability of movement in the last third was greater than that of the middle third of the grasp.

\section{Discussion}

Both the level and variability of motor activity varied systematically before, during, and after oral grasp responses. The level of motor activity before oral grasp responses tended to be lower than baseline, and motor activity after oral grasp responses tended to be higher than baseline. This pattern suggests that oral grasping may be potentiated during periods of low motor activity or inhibited during periods of increased activity. When they observed this phenomenon in the fetal rat, MacLennan et al. (1998) offered the hypothesis that motor quiescence may facilitate processing of sensory information. Both interpretations, however, suggest that fluctuations in motor activity regulate the expression of oral grasp responses.

Alternatively, the oral grasp responses themselves may generate increased motor activity with little effect on the dominant oscillation, the frequency of which remained unchanged during AN presentation (when oral grasping occurred). Short-term activation after AN release would be consistent with the finding that the proportion of high-frequency oscillation increased during nipple presentation. Systematic changes in motor activity also were evident during oral grasp responses. Not only were pups active during oral grasp responses, but they showed increased levels of activity and variability of motor activity during the last third of an oral grasp response.

The level and variability in motor activity covaried and rarely showed differences in their relationship to oral grasp responses. However, in the studies of the late-gestation fetal rat (MacLennan et al., 1998; Reilly et al., 1997), level and variability showed different relationships to oral grasp responses. Differences may be expected because the time scale analyzed in the fetus, $30 \mathrm{~s}$, was much longer than the time scale analyzed in the present study, $5 \mathrm{~s}$ before and after oral grasp responses.

The fact that systematic changes in motor activity were observed during oral grasps of widely different durations suggests that activity during grasps unfolds in a similar pattern that is linked to grasp onset and offset rather than to time. Patterns of cyclicity may change during oral grasping or new movement patterns may be added to the existing motor fluctuations to produce this pattern.

\section{General Discussion}

The present experiment's major findings are that motor activity in the newborn rat is cyclical, that oral responsiveness to an AN by the newborn rat is temporally related to the cycles in motor activity, and that the duration of oral grasp responses increases over time. These findings add a new dimension to the existing knowledge of the array of influences on early suckling behavior in the newborn rat.

\section{Cyclic Organization in Motor Activity}

General motor activity in newborn rat pups is cyclical. This cyclic pattern in activity is evident when the pup is isolated from
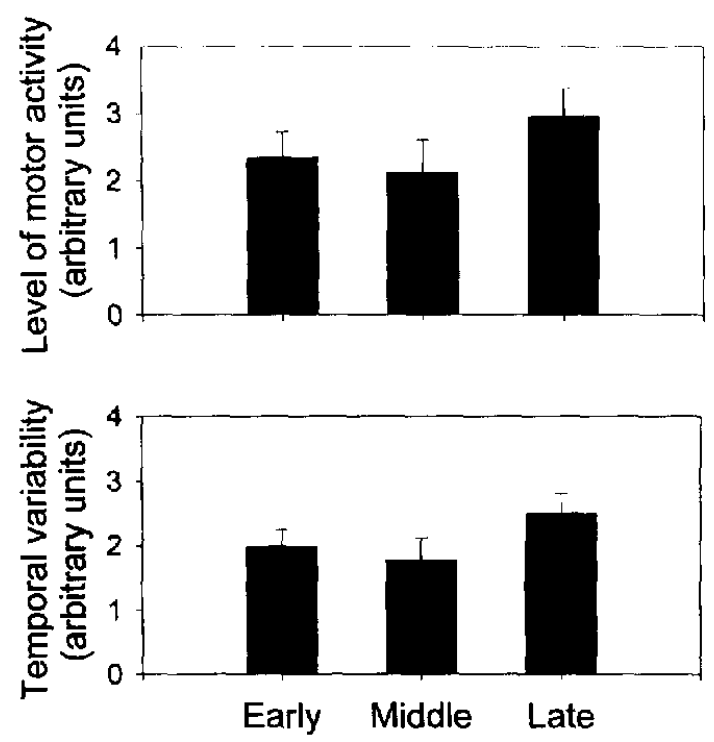

Figure 3. Mean ( $\pm S E$ ) level and variability of motor activity for each third (early, middle, late) of the oral grasp response length. 
its mother and siblings, both of which are known to regulate pup behavior (Hofer, 1991a, 1991b). The cyclic organization is largely unaffected by continuous perioral stimulation with an AN. This suggests that cyclicity in motor activity is a robust feature of the temporal organization in motor activity, and, therefore, it may affect the newborn pup's interactions with its environment (e.g., the expression of suckling behavior at the maternal nipple).

Demonstration of cyclic temporal organization in the newborn rat and previous demonstration of such activity in the fetal rat (Reilly et al., 1997; Smotherman et al., 1988) indicate continuity in this feature of motor organization across the transition from prenatal to postnatal life. This is further evidence of the robustness of the cyclic pattern. Not only was the cyclicity robust, but it also was prevalent. Every rat pup in this study and every fetus in the Reilly et al. (1997) study exhibited cyclic organization in motor activity during both the control and experimental phases of each experiment.

Two differences were noted between the results of the fetal study by Reilly et al. (1997) and the results of the present study. In the fetus, motor activity oscillated faster and the level of motor activity increased during $\mathrm{AN}$ presentation compared with unstimulated controls. Interpretation of these differences must be done cautiously due to substantial methodological differences between the studies. There are three major methodological differences between the fetal study and our newborn study. First, the measures of motor activity are fundamentally different. In the fetal study, an electrode was implanted along the spine in the neck region, whereas in the present study, a sensor was used that did not sample electrical activity, but rather the forces the pup generated by bending the piezoelectric element. Second, motor activity of the fetus in the bath may have a different pattern because the fetus is free floating rather than being constrained against a flat surface by gravity. Third, the Reilly et al. (1997) fetal study used a betweensubjects design, whereas the present study used a within-subject design. These methodological differences alone may explain these few fetal-newborn differences in behavior. For example, the freefloating fetus may show greater activity during nipple presentation due to the relative lack of physical constraint on its range of movement. Alternatively, the behavioral differences might reflect the rapid maturational changes in body size that take place near the end of gestation.

\section{Temporal Relationship Between Fluctuations in Motor Activity and Oral Grasp Responses}

Systematic patterns of motor activity were observed before, during, and after oral grasp responses. First, the onset of an oral grasp response was preceded by a period of relatively low motor activity. Second, pups continued to be active during oral grasps; their activity was comparable to baseline levels but increased before pups released the nipple. Finally, the termination of a grasp response was followed by a relatively high level of motor activity. These systematic patterns of motor activity before, during, and after oral grasp responses suggest that pups initiate and terminate oral grasps at certain times in relation to ongoing fluctuations in motor activity. A similar pattern between the onset of the oral grasp response and cycles in motor activity was found in the late-gestation fetal rat (MacLennan et al., 1998; Reilly et al.,
1997). In MacLennan et al. (1998), oral grasps were more likely to occur when movement was low at the time of nipple presentation.

The increase in level of activity observed in the last third of an oral grasp response may lead to the pup's release of the AN. In a similar fashion, Robertson, Lalley, Bacher, Reilly, and Wood (1995) showed in research with human infants that a certain phase of motor activity cycles (increasing and accelerating) promotes disengagement of visual attention. These findings suggest that cyclic motor activity may regulate infants' interactions with the environment by prompting a change in ongoing behavior (Robertson, 1988).

Although the mechanism of the linkage between motor activity and oral grasping is unclear, one possibility is that cycles of motor activity regulate the timing of pups' responsiveness to biologically relevant stimuli (Reilly et al., 1997). For example, if the pup's activity cycles persist in the presence of its mother and siblings, then the cycles might influence the pup's behavior at the nipple during suckling.

The possibility that cycles in motor activity might regulate oral responsiveness to the AN does not imply that the cycles rigidly determine the timing and duration of the oral grasp responses. Rather, grasps appear more likely to occur at certain times in the fluctuations of motor activity than at others, although grasp onsets may occur (and were observed) at all levels of motor activity. Also, the term regulation does not imply that the stimulus does not elicit responses. It is most likely that the timing of newborn rats' responsiveness to suckling stimuli is an interaction between intrinsic sources of behavioral regulation (e.g., motivation and fluctuations in motor activity) and the influence of the current stimuli (e.g., stimulus intensity).

\section{Increased Duration of Nipple Attachment}

In newborn rat pups' first $10 \mathrm{~min}$ of experience with an $\mathrm{AN}$, the longest oral grasps of the AN were observed in the latter minutes of the nipple presentation. This increase in duration of grasping the nipple might result from grasping experience, the passage of time, or some cumulative effect of the tactile stimulation provided by the nipple. A preliminary comparison to explore possible causes for this increase in duration of oral grasping suggests that the duration of the pup's last oral grasp response is related to the amount of time the pup spent on the nipple before the last grasp (see Figure 4). The greater the amount of time spent on the nipple, the longer the duration of the last oral grasp, $R^{2}=.355, F(1,16)=8.80, p=$ .009 . This relationship suggests that grasping experience promotes later sustained attachment to the nipple.

The importance of suckling experience in promoting continued suckling has been demonstrated by Stoloff, Kenny, Blass, and Hall (1980) in rat pups of 3-5 days of age. They found that both nipple search and attachment were critical for normal suckling on subsequent days of testing. The results of the present study suggest that initial and brief experiences with an AN promote continued expression of oral grasp responses later in the same test session, and they provide a useful starting place for future explorations of the mechanisms of sustained nipple attachment during a pup's first experience with a nipple.

Because pups in the natural suckling context may have to wait minutes while attached to the maternal nipple before the first milk letdown, it is not surprising that a mechanism exists that promotes 


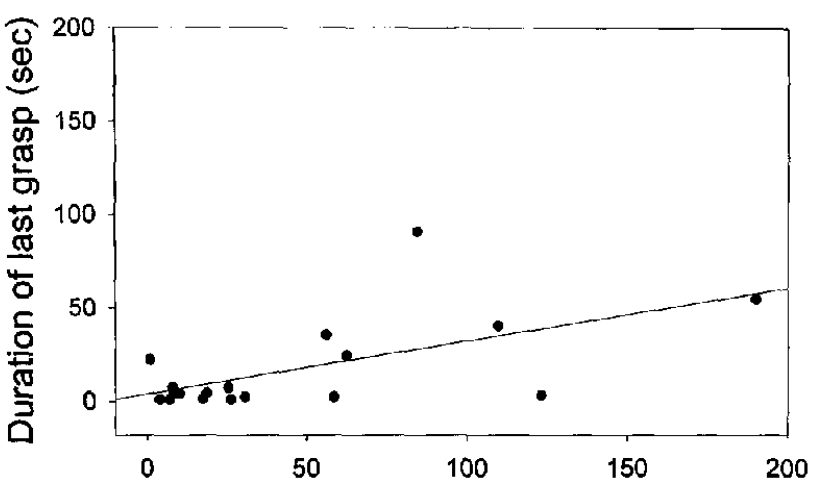

Sum of grasp durations excluding last grasp (sec)

Figure 4. Plot of the duration of the last oral grasp response by the summed durations of all oral grasps except the last grasp.

pups' sustained nipple attachment in the absence of immediate success at obtaining milk. Previous work has demonstrated that pups that have obtained milk from a surrogate nipple show sustained oral grasping when later presented with an empty nipple (Petrov, Varlinskaya, Bregman, \& Smotherman, 1999). The duration of pups' sustained attachment to an AN after experience in obtaining milk from a surrogate nipple is even longer (on the order of minutes) than that observed here. When groups of pups are given equivalent experience with an $\mathrm{AN}$, those that exhibited oral grasps were more likely to show sustained attachment to the AN at reexposure to the AN. In this way, future sustained attachment is dependent on the past performance of an oral grasp response (Petrov et al., 1999). Therefore, the results of the present study suggest that the performance of oral grasping is important for future sustained attachment to the nipple and that increases in the duration of nipple attachment during pups' first experiences with a nipple may occur without milk.

We know that other factors also play a role in the pup's initial nipple attachment. For example, Abel, Ronca, and Alberts (1998) have demonstrated that tactile stimulation (simulated uterine contractions) of the pup at birth is important to subsequent nipple attachment. Also, oral grasping of an AN is increased when the nipple is warm, when the pup is tested in the presence of milk or amniotic fluid odor, and in the presence of a conspecific (Koffman, Petrov, Varlinskaya, \& Smotherman, 1998). The present study suggests that oral grasping of a nipple is important for the development and future expression of sustained attachment to a nipple.

\section{Intrinsic and Extrinsic Influences on Suckling}

Multiple, redundant cues initiate, direct, and sustain behavior in the suckling context (Hall, 1990). The newborn rat pup responds to specific cues from its mother that guide the pup to initiate suckling, but the pup brings to this context an intrinsic source of behavioral regulation that may influence the expression and timing of discrete responses to those cues important for the initiation of suckling. Furthermore, the intrinsic fluctuations in motor activity may provide a mechanism that balances the pup's need to be active to attain some goals (e.g., find a nipple) and be quiet to attain others (e.g., detect and respond to relevant cues; Robertson, 1988; Rapp,
1987). The continuous repetition of the cycles may mean that opportunities for the expression of behavior and detection of relevant cues are frequently repeated. Intrinsic, cyclic patterning of the pup's motor behavior may represent another mechanism to ensure that the pup will continue to behave in ways that maximize the likelihood of growth, development, and survival during early development.

\section{References}

Abel, R. A., Ronca, A. E., \& Alberts, J. R. (1998). Perinatal stimulation facilitates suckling onset in newborn rats. Developmental Psychobiology, 38, 91-99.

Alberts, J. R. (1978). Huddling by rat pups: Group behavioral mechanisms of temperature regulation and energy conservation. Journal of Comparative and Physiological Psychology, 92, 231-245.

Chelazi, L., Biscaldi, M., Corbetta, M., Peru, A., Tassinari, G., \& Berlucchi, G. (1995). Oculomotor activity and visual spatial attention. Behavioural Brain Research, 71, 81-88.

Corner, M. (1977). Sleep and the beginnings of behavior in the animal kingdom - studies of ultradian motility cycles in early life. Progress in Neurobiology, 8, 279-295.

Corner, M. (1978). Spontaneous motor rhythms in early life-phenomenological and neurophysiological aspects. In M. A. Comer, R. E., Baker, N. E. Van del Poll, D. F. Swaab, \& H. M. B. Uylings (Eds.), Progress in brain research: Vol. 48, Maturation of the nervous system (pp. 349-364). New York: Elsevier Scientific.

Eilam, D., \& Smotherman, W. P. (1998). How the neonatal rat gets to the nipple: Common motor modules and their involvement in the expression of early motor behavior. Developmental Psychobiology, 32, 57-66.

Gomez, C., Millan, S., Atienza, M., Aguilar-Bravo, H., Vazque, M., \& Delinte, A. (1998). The gap effect during visual and auditory stimulation using manual responses. Biological Psychology, 47, 77-96.

Hall, W. G. (1990). The ontogeny of ingestive behavior: Changing control of components in the feeding sequence. In E. M. Sticker (Ed.), Handbook of behavioral neurobiology: Vol. 10. Neurobiology of food and fluid intake (pp. 77-123). New York: Plenum Press.

Hamburger, V., Balaban, M., Oppenheim, R., \& Wenger, E. (1966). Periodic motility of normal and spinal chick embryos between 8 and 17 days of incubation. Journal of Experimental Zoology, 159, 1-14.

Hofer, M. A. (1991a). Development, psychobiology. In Encyclopedia of human biology (Vol. 2, pp. 1-11). San Diego, CA: Academic Press.

Hofer, M. A. (1991b). Hidden behavioral regulators in development. In B. J. Carroll \& J. E. Barrett (Eds.), Psychopathology and the brain (pp 113-132). New York: Raven Press.

Holson, R. R., \& Pearce, B. (1992). Principles and pitfalls in the analysis of prenatal treatment effects in multiparous species. Neurotoxicology and Teratology, 14, 221-228.

Jenkins, G. M., \& Watts, D. G. (1968). Spectral analysis and its applications. San Francisco: Holden-Day.

Koffman, D. J., Petrov, E. S., Varlinskaya, E. I., \& Smotherman, W. P. (1998). Thermal, olfactory, and tactile stimuli increase oral grasping of an artificial nipple by the newborn rat. Developmental Psychobiology, 33, 317-326.

MacLennan, B. D., Smotherman, W. P., \& Robertson, S. S. (1998). Variation in motor activity on different time scales and responsiveness to oral stimulation in the rat fetus. Developmental Psychobiology, 33, 125-131.

National Institutes of Health. (1986). Guide for the care and the use of laboratory animals (DHEW Publication No. 86-23). Washington, DC: U.S. Government Printing Office.

Petrov, E. S., Varlinskaya, E. I., Bregman, K., \& Smotherman, W. P. (1999). Sustained attachment in the newborn rat depends on experience 
with the nipple, milk and the performance of oral grasping. Behavioral Neuroscience, 113, 211-221.

Petroy, E. S., Varlinskaya, E. I., \& Smotherman, W. P. (1997). The newborn rat ingests fluids through a surrogate nipple: A new technique for the study of early suckling behavior. Physiological Behavior, 62, $1155-1158$.

Rapp, P. E. (1987). Why are so many biological systems periodic? Progress in Neurobiology, 29, 261-273.

Reilly, J. L., Robertson, S. S., MacLennan, B. D., \& Smotherman, W. P. (1997). Variability in general activity and the expression of complex behavior in the fetal rat (Rattus norvegicus). Behavioral Neuroscience, $111,785-791$.

Robertson, S. (1982). Intrinsic temporal patterning in the spontaneous movement of awake neonates. Child Development, 53, 1016-1021.

Robertson, S. S. (1985). Cyclic motor activity in the human fetus after midgestation. Developmental Psychobiology, 18, 411-419.

Robertson, S. S. (1988). Mechanism and function in cyclicity of spontaneous movement. In W. P. Smotherman \& S. R. Robinson (Eds.), Behavior of the fetus (pp. 77-93). Caldwell, NJ: Telford Press.

Robertson, S. S., \& Bacher, L. F. (1995). Oscillation and chaos in fetal motor activity. In J. P. Lecanuet, W. P. Fifer, N. A. Krasnegor, \& W. P. Smotherman (Eds.), Fetal development: A psychobiological perspective (pp. 169-189). Hillsdale, NJ: Erlbaum.

Robertson, S., Lalley, N., Bacher, L., Reilly, J., \& Wood, J. (1995, March). The dynamics of movement and attention in infants. Poster session presented at the annual meeting of the Society for Research in Child Development, Indianapolis, IN.

Robinson, S. R., Hoeltzel, T. C. M., Cooke, K. M., Umphress, S. M., Murrish, D. E., \& Smotherman, W. P. (1992). Oral capture and grasping of an artificial nipple by rat fetuses. Developmental Psychobiology, 25, 543-555.

Robinson, S. R., \& Smotherman, W. P. (1992). Organization of the stretch response to milk in the rat fetus. Developmental Psychobiology, 25, 33-49.

Robinson, S. R., \& Smotherman, W. P. (1994). Behavioral effects of milk in the fetus. Behavioral Neuroscience, 108, 1139-1149.

Smotherman, W. P., Goffman, D., Petrov, E. S., \& Varlinskaya, E. I. (1997). Oral grasping of a surrogate nipple by the newborn rat. Developmental Psychobiology, 31. 3-17.

Smotherman, W. P., Petrov, E. S., \& Varlinskaya, E. I. (1997). Experimental study of the first suckling episode: Rat pups ingest fluids through the surrogate nipple. Behavioral Neuroscience, 111, 1383-1394.

Smotherman, W. P., \& Robinson, S. R. (1996). The development of behavior before birth. Developmental Psychology, 32, 425-434.

Smotherman, W. P., Robinson, S. R., \& Robertson, S. S. (1988). Cyclic motor activity in the fetal rat (Rattus norvegicus). Journal of Comparative Psychology, 102, 78-82.

Stoloff, M. L., Kenny, J. T., Blass, E. M., \& Hall, W. G. (1980). The role of experience in suckling maintenance in albino rats. Journal of Comparative and Physiological Psychology, 94, 847-856.

Winer, B. J. (1971). Statistical principles in experimental design (3rd ed.). New York: McGraw-Hall.

Received May 28, 1999

Revision received October 11, 1999

Accepted January 20, 2000 